\title{
High-Resolution 3D Imaging of Virus Infections in Solvent-Cleared Organs: Novel Insights into Virus Replication and Tropism In Vivo ${ }^{\dagger}$
}

\author{
Luca Zaeck, Madlin Potratz, Antonia Klein, Conrad M. Freuling, Thomas Müller \\ and Stefan Finke *
}

Institute of Molecular Virology and Cell Biology, Friedrich-Loeffler-Institut-Federal Research Institute for Animal Health, 17493 Greifswald - Isle of Riems, Germany; luca.zaeck@fli.de (L.Z.); madlin.potratz@fli.de (M.P.); antonia.klein@fli.de (A.K.); conrad.freuling@fli.de (C.M.F.); thomas.mueller@fli.de (T.M.)

* Correspondence: stefan.finke@fli.de (S.F.)

† Presented at Viruses 2020-Novel Concepts in Virology, Barcelona, Spain, 5-7 February 2020.

Published: 17 June 2020

\begin{abstract}
The visualization of infection processes in relevant tissues and organs using microscopy methods reveals a unique link between the distribution, tropism, and abundance of pathogens and the physiological structure of the respective organ. To dissect virus replication and the host reaction in vivo at both a global and a single-cell level, conventional 2D imaging approaches can only provide limited insight. However, pathological studies of infected organ material are still mostly restricted to the immunostaining of thin sections from paraffin-embedded or frozen samples. While the 3D analysis of large tissue volumes is possible via laborious serial sectioning, a variety of problems and artifacts remain. Modern immunostaining-compatible tissue clearing techniques allow for the seamless 3D visualization of infection sites in optically cleared thick tissues sections or even entire organs. Benefiting from pure optical slicing, this approach enables the acquisition of multicolor high-volume 3D image stacks for coherent qualitative and quantitative analyses of the infection and its surrounding cellular environment. Here, we demonstrate the utility and power of this methodology by visualizing virus infections in different target tissues. For instance, we reconstructed the cellular context of rabies virus infection sites in mouse brain tissue, allowing a thorough investigation and quantitative analysis of rabies virus cell tropism. The systematic comparison of different rabies viruses with varying pathogenicity revealed a remarkable difference for highly virulent street rabies viruses and attenuated lab strains. While the virus protein expression was readily detectable at a comparable level in both neurons and non-neuronal glial cells from brains of mice infected with street rabies viruses, it was virtually absent in glial cells of lab strain-infected mice. These data provide novel and detailed insights into the pathogenesis of virus infections and substantially contribute to an improved understanding of virus-host interactions in vivo.
\end{abstract}

Keywords: tissue optical clearing; 3D immunofluorescence; $\mathrm{uDISCO}$; rabies virus; astrocyte infection

(C) 2020 by the authors. Licensee MDPI, Basel, Switzerland. This article is an open access article distributed under the terms and conditions of the Creative Commons Attribution (CC BY) license (http://creativecommons.org/licenses/by/4.0/). 